J. Andersen (ed.), Reports on Astronomy, Vol. XXIVA, 21-31.

(C)2000 IAU. Printed in the United States of America.

\title{
COMMISSION 8: POSITIONAL ASTRONOMY
}

\section{(ASTRONOMIE DE POSITION)}

\section{PRESIDENT: H. Schwan \\ VICE-PRESIDENT: Wenjing Jin}

ORGANIZING COMMITTEE: T.E. Corbin, J. Kovalevsky, J.A. Lopez,

\author{
L.V. Morrison, F. Noel, G. Pinigin, D. Polojentsev, R. Stone \& J. Xu
}

\section{INTRODUCTION}

The present triennial commission report embraces mainly activities in wide angle, optical astrometry. With the successful development and application of new techniques from Earth (e.g. optical interferometry, CCD's) and space (Hipparcos mission and new projects) the sub-division between Commissions 8 (Positional Astrometry) and 24 (Photographic Astrometry) has become questionable. During the GA at Kyoto in 1997 all steps for a merger of both commissions have been taken. The final merging will take place at the forthcoming GA in Manchester. For a more complete overview on astrometrical work done in the past triennium the reader should also take notice of the report of Commission 24 .

\section{SCIENTIFIC HIGHLIGHTS}

Based on resolutions adopted during the GA at Kyoto the International Celestial Reference System (ICRS) has come in official use starting on 1 January 1998 with the Hipparcos catalogue being its materialization in optical wavelengths. Programs for densifying and extending this frame to fainter magnitudes are under way. Much progress and first successful observations in optical interferometry have been carried out, space missions are planned.

\section{INSTRUMENTATION AND CATALOGUES}

\subsection{Argentina}

The San Fernando Automatic Meridian Circle (CMASF) of the Real Instituto y Observatorio de la Armada (ROA) in Spain has been operating at the Carlos Ulico Cesco Astronomical Station (CUC) of the Observatorio Astronomico Felix Aguilar (OAFA) since June 1996. It is operated jointly by the ROA and the OAFA. The costs of the movement of the CMASF from Spain to Argentina and the installation in the CUC have been shared between the Ministerio de Defensa, the Ministerio de Educación y Cultura of Spain and the Universidad de San Juan of Argentina. The CMASF is provided with a scanning micrometer similar to that installed until a year ago in the Carlsberg Automatic Meridian Circle in La Palma. Since November 1997 a program of observations of faint reference stars in the south hemisphere is in progress. A list of Solar System objects is being observed regularly. It is planned to install a CCD camera borrowed by the ROA from the Copenhagen University Observatory in October 1999. The Meridian Catalogue with the results of the observations carried out since October 1997 to October 1999 will be published in 2000.

A covariance matrix for total least squares with heteroscedastic data has been derived and applied to 1140 observations of Pluto (Astron. J. 117, p. 1942, 1999). 


\subsection{Chile}

The Danjon Astrolabe Project at Santiago, a joint program between ESO and Universidad de Chile, is in progress since 1965. The systematic differences Astrolabe-FK5 between $-70^{\circ}$ and $+20^{\circ}$ were evaluated using observations from $1990-1994$ (A \& AS 124, 153). A program of solar astrometry is in progress since 1990. More than 4400 observations of solar borders have been made showing a dependence of the observed Sun semidiameter on the solar cycle (A \& AS 132, 195) and the heliographic latitude (A \& A 343, 1001).

Automation of the astrolabe using a CCD camera was stopped because of the poor results caused by light scattering coming from the peculiar optical structure of the Danjon astrolabe.

\subsection{China(PR)}

This report covers scientific activities done under the Committee of Catalogues \& Astronomical Constants, which is part of the Chinese Astronomical Society.

The activities with the Photoelectic Astrolabe Type III at the Beijing observatory and with the Horizontal Meridian Circle at Shaanxi observatory were discontinued for scientific and economical reasons.

The prototype optical interferometer has been further developed by Shaanxi Astronomical Observatory and Nanjing Astronomical Instrument Research Center; fringes of artificial light (bandwidth $500 \AA$ ) were observed in 1996 (Publ. Shaanxi Astr. Obs. 1, 65).

Photoelectric astrolabes Type Mark I and Mark II were operated by Yunnan, Shaanxi and Beijing Observatories. On the basis of observations made by Beijing Observatory at San Juan (Argentina) the star catalogue CPASJ1 of 2980 stars within $-3^{\circ}$ to $-60^{\circ}$ and from magnitudes 2.0 to 11.3 was constructed. The mean precision is 0 " 046 at the mean epoch 1993.6 (A\&AS 118,1). The radio stars contained in this catalogue were improved by new observations. A catalogue of 69 radio stars with a precision of 0.0022 in RA and $0 !^{\prime \prime} 035$ in Dec has been compiled (Publ. Beijing Astr. Obs. 32, 87). Double transit observations made in 1995 and 1996 have allowed to correct the positions of Uranus and Neptune.

The photoelectric astrolabe catalogue of Yunnan Observatory was compiled from observations made 1987 to 1992 . Positions of 53 FK5, 70 FK5 Extension and 486 GC stars are given for J2000.0 with an internal precision of \pm 0 " 046 in RA and \pm 0 " 059 in Dec at the mean epoch 1989.51 (A\&AS 118, 239). Optical positions of 44 radio stars will soon be published (Hu et al. 1999, in press in AJ).

The photoelectric astrolabe of Shaanxi Astronomical Observatory has been working in Irkutsk, Russia since Nov. 1995. From these observations the EOP in Russia are determined. An astrolabe catalogue of 200 stars was compiled (including as many radio stars as possible) which is the first one without a blind zone in declination. The precision obtained is \pm 0.0023 in RA and \pm 0.031 in Dec. (Publ. Shaanxi Astr. Obs. 21, No. 1, 54).

A joint project between Shanghai Observatory and Mykolayiv Observatory is being carried out for determining precise optical positions of secondary reference stars around about 70 extragalactic radio sources using the 1.56 telescope at Shanghai and the fully automatic meridian circle at Mykolayiv.

A combined catalogue of the optical positions of about 510 extragalactic radio sources was constructed on the basis of 28 individual catalogues. The precision of the included 56 primary sources is 0.09 . The rotation angles between the optical and the radio frame were determined (A\&AS 120, 201).

A proper motion catalogue containing 1663 stars with 996 stars being members of the Orion Nebular Cluster was constructed from four high-precision individual proper motion catalogues and new observations (Annals of Shanghai Obs. Academica Sinica 17, 223).

A new approach for compiling extragalactic radio source catalogues was proposed based on the arc length difference between primary sources. The stability of orientation of the combined catalogue is better than 0.1 mas (Astr. Astrophys. Trans. 13, 1). 
A formula modelling refraction at high zenith distances was derived (AJ 112, 1312)

\subsection{Denmark, Great Britain \& Spain}

The Carlsberg Meridian Telescope (CMT) continued to be operated at the international observatory of the Roque de los Muchachos on the island of La Palma in the Canaries by the three participating institutions: Copenhagen University Observatory, the Royal Greenwich Observatory and the Real Observatorio y Instituto de la Armada en San Fernando.

All the observations made with the scanning-slit micrometer from May 1984 until its replacement by a CCD camera in June 1998 were issued in May 1999 as a composite catalogue on a CD-Rom. This CD-Rom comprises Carlsberg Meridian Catalogues (CMC) 1 to 11 and contains 180812 positions and magnitudes of 176591 stars north of declination $-40^{\circ}, 155005$ proper motions, and 21944 positions and magnitudes of 184 Solar System objects. All the positions are referred to the International Celestial Reference Frame. The mean error of a catalogue position for a star of magnitude $V=13$ in the zenith is 0.10 in CMC1-4, improving to $0{ }^{\prime \prime} 07$ in CMC10-11. The accuracy in magnitude is 0.05 mag in $\mathrm{CMC} 4-10$, improving to $0.03 \mathrm{mag}$ in CMC11. The mean error of the proper motions derived by combining the positions in CMC1-11 with those at earlier epochs is typically in the range 0."003 to 0." 004 per year. About 33700 stars were observed with the primary purpose of determining their proper motions. The magnitude limit of the catalogue is $V=15.4$ and most of the stars are fainter than the limit of the Tycho Catalogue. Special lists of reference stars include stars in the range $10<V<13$ within 20arcmin of VeronCetty galaxies. The catalogue also includes the following programmes of stars of $12<\mathrm{V}<14$ with formerly poor positions: Gliese catalogue of nearby stars (CNS3), high proper motion stars (NLTT) and variable stars (GCVS).

The Carlsberg observations of the major planets were used to check the accuracy of JPL DE405 [A\&AS, 132, 381-386 (1998)]

In June 1998 the photoelectric scanning-slit micrometer was replaced by a prototype CCD camera. During March 1999 a new custom-built camera using a Kodak $2060 \times$ $2048 \mu \mathrm{m}$ pixel size (0!'7) was installed. This has been used with a Sloan $r^{\prime}$ filter to begin an astrometric survey of the N. hemisphere down to $r$; 17.0 using the ACT Reference Catalog to define the reference frame.

The telescope has been operated remotely via the Internet starting in April 1997.

\subsection{France}

Most activities concern the exploitation of the Hipparcos catalogue for astrophysical investigation (see Comm. 24).

\subsection{Germany}

Work continued on the data base ARIGFH at the Astronomisches Rechen-Institut (ARI). The aim is to collect all relevant astrometric data on stellar positions and proper motions in a comprehensive data bank. About 10 millions of observations published in about 1400 individual catalogues are presently identified with a large master catalogue.

At ARI work is in progress to combine the Hipparcos catalogue with various ground-based catalogues such as the FK5 or GC. The aim is first to derive improved proper motions and second to give information on possible undetected astrometric binaries. Such stars may show up in the differences between the ground based and the Hipparcos proper motions (so-called $\Delta \mu$ binaries). Probability value for their possible duplicity are given. The combination of Hipparcos with 878 single-star candidates in the FK5 is completed and published as the "Sixth Catalogue of Fundamental Stars (FK6), Part I" (Veröff. Astron. Rechen-Inst. No. 35). Various solutions (modes) for each star are given depending on different assumptions concerning the so-called cosmic errors. For the theoretical background reference is made to "Principles of Statistical Astrometry" (A \& A 325, 367; A \& A 346, 675; A \& A 347, 
1046). The overall precision of the FK6(Part I) in the single-star mode is 0.54 mas at the central epoch 1991.22 and 0.35 mas/yr for the proper motions.

\subsection{Japan}

A long-baseline optical/IR interferometer for astrometry and high-resolution imaging is being developed at National Astronomical Observatory, Japan (NAOJ) since 1996. The first phase instrument (MIRA-I) consisted of two Coude telescopes aligned along a $4 \mathrm{~m} \mathrm{~N}-\mathrm{S}$ baseline and one optical bench in between. A number of optical components working at the red band $(800 \mathrm{~nm})$ were arranged on the optical bench. First stellar fringes with MIRAI were obtained in June 1998 for Vega with $6 \mathrm{~cm}$ aperture at each telescope. Changes in the optical components have improved the quality and efficiency of fringe detection (MIRA-I.1). Fringes of nine stars were observed, the baseline vector and the apparent diameter of Aldebaran were determined, fringe tracking experiments were made, the powers of atmospheric fluctuations of the angle of arrival were measured, the limiting magnitude reachable at the Mitaka was estimated. Since April 1999 a two-element, long baseline $(30 \mathrm{~m})$ interferometer of $30 \mathrm{~cm}$ siderostat aperture (MIRA-I.2) is scheduled for construction within two years (Proc. of SPIE, 3350, 202-217, 1998).

Observations of 6649 stars made from 1990 - 1993 with the Tokyo PMC were published (Publ. NAOJ 5, 1). Variations of the solar-radius over a solar cycle were observed with the Tokyo PMC (IAU Coll. 165, 551). The Tokyo PMC and CCD meridian circle are still in operation for observations of major planets and their satellites. The strip scanning of the sphere with the CCD meridian circle is in progress for observations of faint reference stars. The regular observational activities with the meridian circles will be continued till the end of March 2001.

\subsection{Russia}

At the Pulkovo Observatory observations of star declinations made with the Zverev Photographic Vertical Circle (PhVC) were finished successfully. During 1987-1998 more than 13000 fundamental observations of FK5 stars were made, reduced and published as FVK96 catalogue (Izvestia GAO, No. 213,48). The catalogue precision is 0.1 . A new receiving device was installed to PhVC including the camera ST-6 with a mechanism of fixation and regulation of its position, a shuttle with 2-petal, and a mechanism for remote filter change. The instrument was renamed: PMT (Pulkovo Meridian Telescope) of M.S. Zverev. Experimental CCD observations showed that a limit of $15.7 \mathrm{mag}$ can be achieved. The mean observational errors are from $0{ }^{\prime \prime} 06$ to $0{ }^{\prime \prime} 25$ depending on the object's magnitude (Izvestia GAO, No. 213, p.217).

At the Kislovodsk Mountain Station of the Pulkovo Observatory regular observations of the Sun, Mercury, Venus and Mars were carried out. With the Ertel-Struve Transit Instrument 292 observations of the Sun and planets were made with a mean error of 0.050 (VINITI No. 2363-B98, 1998), and 408 observations of the Sun and planets were made with the Ertel-Struve Vertical with a mean error 0"40 (VINIT 2362-B98,1998).

The Meridian Automatic Horizontal Instrument of the name of Suharev (MAHIS) was mounted in its pavilion at the Pulkovo Observatory. Experimental star observations have been started using a CCD micrometer; its mosaic variant is being tested. The field of view is 40 arcminutes. A computer program for running the telescope and its CCD micrometer was developed.

Observations of Uranus ( $~ 3000$ observations), Neptune (8000) and Pluto (1362) made at different observatories were analyzed by a global treatment and reduced to the FK5 system. Corrections to the planetary masses were taken into account. The orbital improvement was made by a differential method. The spectral analysis of the residuals showed no indication for a possible "X-planet" (Preprint of GAO, Lab.Phot., No. 7, p. 76, 1996). 
Photoelectric observations of lunar occultations of 63 stars were made at Engelhardt Astronomical Observatory (Kazan) from 1984 through 1997 with a precision of a few milliseconds, and diameters of 12 stars have been determined (AJ 116, 1504).

\subsection{Ukraine}

At the Mykolayiv Astronomical Observatory Solar system objects were observed with the zone astrograph $(\mathrm{D}=120, \mathrm{~F}=2044 \mathrm{~mm}$, plate $50 \times 50)$ : Mars (15 observations), major planets (82), minor planets (88), Jupiter satellites (189), Saturn satellites (26). Observations of Jupiter's Galilean Satellites made from 1962 through 1996 were reduced to the ICRS system and catalogue positions were obtained with a precision of 0. " 1 . Similarly 2463 observations of 19 minor planets made from 1961 until 1997 were reduced to the ICRS system with a precision ranging from $0 .{ }^{\prime \prime} 12$ to $0 .{ }^{\prime \prime} 18$, dependending on the magnitude.

Finished were observations of secondary reference stars made with the CCD Axial meridian circle in fields around 190 extragalactic radio sources north of $-20^{\circ}$ and from 12.0 to 14.5 th mag. About 100,000 observations of 20,000 stars (including Hipparcos stars) were made; GSC star positions were reduced to the Hipparcos system. The variations of the instrumental system were not larger than $0{ }^{\prime \prime} 02$. The precision of one observation for Hipparcos stars was $0{ }^{\prime \prime} 11 \mathrm{sec} 0.65 z$ and about $0.13-0 !^{\prime \prime} 20$ for the fainter GSC stars. A catalogue precision of about $0.03-0{ }^{\prime \prime} 05$ is expected. The data processing is being continued. In the beginning of 1999 a new CCD $\left(1040 \times 1160,16 \times 16 \mu m\right.$ pixel size, FOV $\left.22 \times 24^{\prime}\right)$ was attached to the AMC and preparations have started for an installation of a similar CCD in the zone astrograph.

At the Main Astronomical Observatory of Ukrainian Academy of Sciences the creation of an infrared reference catalogue was continued. This catalogue should represent the ICRS system in the IR and it should serve as the reference frame for ground-based differential observations (in the IR) using array detectors with fields of view of about 15-20 arcminutes. The following major subtasks were carried out: 1) A preliminary catalog of infrared sources was created (Baltic Astronomy, 6, 344); 2) A list of 232 radio stars with probable counterparts in the infrared was compiled and proposed for new photometric and spectral ground-based or space observations (Proceedings of the Journees 1998, in press; a preliminary version can be obtained by anonymous $\mathrm{ftp}: / / \mathrm{ftp} 2$. mao.kiev).

For maintaining "The Planets" data base, observations of the Sun and seven large planets from 1960.0 to 1995 were included. Updating and analysis is made in cooperation with the Institute of Astronomy (INASAN) in Moscow (Baltic Astronomy 6, 255)

At the Astronomical Observatory of the University of Kyiv the arc length method was used to derive the combined radio catalogues RSC(GAOUA) $98 \mathrm{C} 01$ and RSC(GAOUA)99 $\mathrm{C}$ 01, based on individual catalogues submitted to the IERS Central Bureau in 1997 and 1998. The axes are aligned to the ICRF with an accuracy of 0.02 mas.

The Kyiv Meridian Axial Circle (MAK) was equipped with a new CCD micrometer, manufactured at Mykolayiv Observatory.

\subsection{USA}

Final reductions were completed on the Pole-to-pole project with the resulting catalog called the W2J00. Observations were made between 1985 and 1996 using transit circles located in Washington DC, USA and near Blenheim, New Zealand. The W2J00 was linked differentially to the ICRF using star positions from the Hipparcos catalog. The two transit circles are now inactive.

The period saw the completion of a functioning sub-set of the astrometric array at the Navy Prototype Optical Interferometer (NPOI). The environmental chambers for the baseline metrology system at all four astrometric siderostat stations were completed and temperature stabilized to the required level $\left(0.2^{\circ} \mathrm{C}\right.$ peak-peak over night). All of the major optical and electronics components for the baseline metrology at three of the four stations were installed and tested. There is now fully functioning metrology to measure the sidero- 
stat pivot motions with respect to the reference tables at these three stations, as well as metrology (Aoptical anchors) to measure the reference tables positions with respect to the local bedrock $\sim 8 \mathrm{~m}$ below the site. The data analysis software for the baseline metrology was completed during the period and extensively tested. The software can remove the occasional discontinuities in the raw data caused by coating imperfections in the siderostat retroreflectors, and has been shown to reproduce externally applied displacements of the siderostat pivots at the level of $\sim 1$ micron rms.

Preliminary wide-angle observations with the 3-element NPOI array began in 1998. To date, all sky astrometric solutions have only achieved 20-30 mas formal errors (one night) due to a combination of drifts in the internal optical paths of the vacuum feed system between the siderostats and the lab, and less than fully adequate dispersion correction of the raw stellar fringe data. The former problem is being addressed through extensive mechanical improvements in the structures holding the optical components of the feed system and through thermal control. The second problem is addressed through improvements in the fringe analysis algorithms allowing coherent integration of many $2 \mathrm{~ms}$ fringe frames in order to increase the signal-to-noise of the detected fringes. This allows a more accurate dispersion correction of the observed stellar delays to their equivalent vacuum values.

Medium angle $\left(\sim 10^{\circ}\right)$ relative astrometry has also been attempted with the astrometric array of the NPOI. Initial analyses show encouraging results, with formal errors better than 20 mas indicated.

The NPOI has also continued its very successful observational programs for binary stars, stellar diameters, and imaging experiments. The visual orbits of several spectroscopic binaries have been completed, including systems with semi-major axes as small as $\sim 3$ mas (o Leonis). Angular diameters for several dozen stars (including a bright cepheid), accurate to 1-3\% have been determined. The NPOI also obtained some of the first direct measurements of stellar limb darkened diameters, other than those for $\alpha$ Bootis and the Sun, and produced some of the first synthetic aperture maps ever obtained with a multi-element long baseline interferometer (Mizar).

\subsection{Yugoslavia}

Ivan Pakvor informs that the Belgrade Meridian Circle (BMC) was completely destroyed on May 10, 1999 during the Yugoslavia conflict. The BMC was one of the three fundamental instruments (Transit Instr. \& Vertical Circle) with the same aperture and focal distance $(190 / 2578 \mathrm{~mm})$ that existed at Belgrade Observatory. Observations carried out since 1970 with BMC have contributed to the International References Frame.

\section{REFERENCE FRAME, EXTRAGALACTIC LINK}

Feissel and Mignard have described the meaning and the consequences arising from the adoption of the International Reference System ICRS (A\&A 331, L33).

USA: Numerous VLBI experiments were proposed, scheduled, and observed for the purpose of maintaining and improving the accuracy of the International Celestial Reference Frame (ICRF). The densification of the ICRF frame, and the radio/optical link are ongoing concerns. The US Naval Observatory (USNO) produced a new list of possible ICRF sources from data obtained from the NRAO sky survey. Additions to the radio star list for common NPOI/VLBI observing were also identified by searching several large databases and catalogs of radio stars. These will assist in strengthening the link of the radio and optical frames. The list consists of radio stars with at least one confirmed observation of flux over $5 \mathrm{mJy}$, and brighter than visual magnitude 10 . There are now 140 candidate stars. Further observation and investigation will determine which are most suitable.

Reductions of CCD observations of optical counterparts of ICRF sources obtained 1994-1997 at Kitt Peak and Cerro Tololo were completed using photographic reference star data provided by the Hamburg Observatory. A paper (Zacharias, N et al. AJ Nov. 1999) 
concludes this decade long, global project, providing optical positions of 327 sources on the Hipparcos system with an average accuracy of 50 mas per source and 4 mas error for the frame alignment. Updated finding charts of these sources appear on the USNO web site.

In 1997 long-term status was granted for the extragalactic link program of the UCAC project. In 7 observing runs at the CTIO 0.9-meter telescope data were obtained for about 200 sources with parallel observing at the CCD Astrograph to provide reference stars at the same epoch, using the same spectral bandpass (579-642 nm). All raw data reductions and image checking were completed. The collaboration with colleagues from Brazil continued with additional observations of a sample of the ICRF sources at the LAN 1.6-meter telescope.

Several hundred of $\mathrm{S}$ and $\mathrm{X}$ band images of ICRF sources were added to the Radio Reference Frame Image Database (RRFID), primarily (but not entirely) as a result of processing 6 additional RDV experiments. The images were made available on-line (http://www.usno.navy.mil/RRFID). The RRFID is now composed of 2223 images of 426 sources and contains one or more images of nearly all ICRF sources north of $-20^{\circ}$.

An agreement was negotiated between USNO and the Australian CSIRO for a 5-year joint observing program to strengthen the ICRF in the Southern Hemisphere. There are two parts to the 5-year program: the first is to image about 200 sources south of $-20^{\circ}$ while the second is to make multi-epoch astrometric observations of about 150 sources.

A manuscript "VLBA Observations of Radio Reference Frame Sources. III. Astrometric Suitability of an Additional 225 Sources,** was prepared and submitted to the Astrophys. J. It reports intrinsic structure information for 225 ICRF sources and estimates the effects of intrinsic structure on the accuracy and stability of the ICRF sources.

ARGENTINA: Branham reports on a study of the FK5 equinox and equator performed on the basis of about 11700 observations of 21 minor planets made from 1857 - 1993. (Rev. Mex. Astron. Astrophys. 34, 3, 1998).

RUSSIA: Fomin (Pulkovo) reports on a more precise estimation of the FK5 zero point and its secular change based on meridian lunar observation at Greenwich in the 19th century.

CHINA: At Shaanxi observatory the orientation between various reference frames was studied including IERS, DE200, Hipparcos (H37), ICRF and the FK5 (Publ. Shaanxi Obs. 20, 83 and Acta Astr. Sinica 41, 107).

A radio reference frame was constructed based on VLBI observations. Structure corrections for some radio sources were modelled by a two-component model. For 1015+018 changes in the radio source positions, the base length and EOPs of the order of centimeters and sub-milliarcseconds were found (Publ. Shaanxi Astron. Obs. 21, 54).

Long series of optical observations of 43 extragalactic radio sources were used to derive the corrections to the precessional constant (A\&A 318,653).

Observations of asteroids with the LLMC were discussed with the aim to determine the zero point of the celestial reference frame (Publ. Yunnan Obs. 65, No. 2, p. 27).

A study of the general relativistic effects in the definition of the ecliptic showed that these effects are insignificant at present and in the near future (A\&A 333, 374).

UKRAINE: At the Main Astronomical Observatory of Ukrainian Academy of Sciences a more rigorous geometrical model was developed for solving the problem of fitting the stellar and planetary reference frames using the observations of the Solar system bodies. The imperfection of the Earth's motion theory and observation reductions are proposed as an explanation for the non-precessional motion of the equinox.

\section{SPACE ASTROMETRY}

The USNO successfully proposed a program in 1998 for the preparation of a grid star list for the Space Interferometry Mission (SIM). The critical requirement is for the stars of the list to be as free as possible of companions. To that end, the USNO has selected 7,200 stars of visual, apparent magnitude 6.5 to 8.5 that are of class $\mathrm{V}$ and types A5 to K5. The 
survey of the list has begun with speckle interferometers. A CCD camera for detecting wide doubles and a Fourier Transform Spectrometer (FTS) for detecting low mass companions have been under development during the first year of the program. It is expected that surveys of the list will begin next year with the CCD camera, the FTS and the USNO optical interferometer. The double star program has been reported to Commission 26.

The US Naval Observatory has formed a team with the Naval Research Laboratory, Smithsonian Astrophysical Observatory and Lockheed Martin to propose an astrometric satellite mission be funded by NASA as part of their MIDEX series. The goal is to observe 40 million stars between $\mathrm{m}(\mathrm{v}) 7$ to 15 , with position, proper motion, and parallax accuracies of 50 micro-arcsec, 50 micro-arcsec/yr and 50 micro-arcsec, respectively. The proposal for this satellite, dubbed Full-Sky Astrometric Mapping Explorer (FAME), was accepted for the Phase A Concept Study. Two of the five proposed missions are expected to be fully funded; the announcement of which two should be made in the Autumn of 1999.

Lindegren (Lund Observatory, Sweden) reports that the GAIA project has been under study during 1997-99 by the ESA as a candidate for a cornerstone mission with possible launch in 2009. GAIA will rely on the proven principles of ESA's Hipparcos mission to create a precise kinematic and photometric mapping of more than a billion stars throughout the Galaxy and beyond. Virtually all pointlike objects down to the 20th magnitude can be measured, with a precision of 0.01 mas at $15 \mathrm{mag}$ and 0.2 mas at $20 \mathrm{mag}$. Multi-epoch, multi-colour broad-band photometry is obtained for all the stars, radial velocities and more detailed (medium-band) photometry for many of them. Observation of quasars provides a direct link with the extragalactic reference frame to better than $1 \mu \mathrm{as} / \mathrm{yr}$. The main science goal is to clarify the origin and history of our Galaxy by providing a massive census of the Galactic stellar populations, with the precision necessary to test formation scenarios and unravel the subsequent evolution. Additional goals include studies of extra-solar planetary systems, stellar physics, General Relativity and the Solar System. The continuously rotating satellite employs two astrometric telescopes for wide-angle measurements through large CCD mosaics operated in drift-scanning mode. A third telescope is dedicated to radial velocities and medium-band photometry. The technical studies have demonstrated that the project is feasible and fully consistent with the time scale and budgetary constraints of an ESA cornerstone mission. (Main web site: http://astro.estec.esa.nl/GAIA/).

Röser at Heidelberg reports on the project of a small satellite mission named DIVA (Double Interferometer for Visual Astrometry). DIVA is to perform a complete sky survey down to about $\mathrm{V}=15$. It will measure the parallaxes, positions, proper motions, magnitudes and colours of some 40 million stars. Spectrophotometry between 400 and $1000 \mathrm{~nm}$ with a spectral resolution of about 20 will be obtained for all objects brighter than $V=13$. Its performance can be characterized by adopting Hipparcos as benchmark: DIVA will observe 300 times as many stars as Hipparcos, it will measure Hipparcos-stars with 5 times better accuracy, and will reach the same accuracy as Hipparcos at stars five magnitudes fainter. A feasibility study has been successfully completed in 1998 . If funded, DIVA could be launched in 2003; after 2 years of mesurements and another 2 years of data reduction, results could be available around 2007. The DIVA web address is http://www.aip.de/groups/diva.

Frink and Quirrenbach (San Diego), Röser (Heidelberg) and Schilbach (Potsdam) have started work on the selection of suitable candidate stars for the astrometric grid of the Space Interferometry Mission (SIM). As a first approach, $\mathrm{K}$ giants from the Hipparcos catalogue have been checked for possible companions. Information from the Hipparcos catalogue itself as well as a comparison with ACT/TRC has been used to eliminate binaries.

At Pulkovo Observatory the work for modelling the observations made onboard of the STRUVE Space Astrometric Project with a precision of 0.3 mas is very near to completion. Based on a study of 300 publications with solar observations in the 20th Century a space astrometric device for observing the Sun on the star background was proposed (D. Polojentsev: Les Journees 1999, IX Lohrmann Kolloquium, in press). 


\section{WORKING GROUPS}

The WG on re-measurement of Astrographic Plates is sponsored jointly by Commissions 8 and 24 and the report is in Commission 24.

ASTROLABES (F. Chollet, Chairman). The report concerns astrolabe groups which continue (or begin) to do astrometry with astrolabe and maintain connection with the WG. These groups are not numerous. Their observing programs have changed since 1987.

The use of CCD cameras to replace the observer becomes widespread since the first tests done by F. Laclare during the 1990's, for solar diameter evaluations.

The astrolabe activities concern now primarily instrumentation and observations of selected objects with the aim to contribute to the development of the new network of fundamental astrometry. The observing programmes are devoted to those objects which have not been observed from space and which are expected to be of importance for defining the connection between different celestial reference frames.

It is important to note that with merely a few minor changes the modern astrolabes have become able to contribute to projects which are entirely different from those for which the WG "Astrolabes" had originally been created. The main goal of this report is therefore to describe the new developments with the consequence to propose the replacement the WG by a new one devoted to those tasks which are the new working field of the astrolabes.

Instrumentation: The astrolabes at the "Observatoire de la Cote d'Azur/CERGA" (OCA/CERGA) in France, at San Fernando in Spain, and at Saõ Paulo and Rio in Brazil are now equipped with CCD video cameras which allow one to observe the Sun for determinig the solar diameter and its variations. The instruments are at present only semiautomated, except for the new full-automated DoRaySol instrument (OCA/CERGA). In 1999 the astrolabe of Malatya (Turkey) was moved to Antalya and it was equipped with a CCD video camera. In China photoelectric astrolabes are used in cooperation with Russia (Irkoutsk) and Argentina (San Juan). The Chinese astrolabes are the only instruments working on star catalogues.

The use of CCD implies changes in the original design of the Danjon astrolabe. At present the mentioned instruments, except DoRaySol, San Fernando and China instruments, continue to use the Wollaston prism in a fixed position, in order to correct for the origin optical defect of the Claude astrolabe. Removal of this device implies to use alternatively new optical systems as those studied now in Poznan and Bucarest or to use a full pupil.

Network and scientific program: The network of the new astrometric instruments consists at present of the following five stations, which participate to solar observations using CCD cameras: CERGA, Rio de Janeiro, Sao Paulo, San Fernando and, since June 1999, Antalya. Since 1990 Santiago is also participating but, for the moment, in visual mode only. Projects for star observations are under way in Poznan and Bucarest, and for solar and star observations in the north of Brazil. The equipment of astrolabes with CCD devices has caused some problems for star observations since an exposure time as short as possible is needed in order to have precise positions on the CCD frame. Present CCD devices are not sensitive enough for observations of stars fainter than about 3.5 to $4 \mathrm{mag}$, depending on the place and the state of the instrument. Except for tests done at Poznan and Bucarest, no regular CCD star observations are done. However new light intensifiers have been developed which could be used in combination with CCD cameras, thus permitting to increase the magnitude limit to 9 or even more. Testing of these new devices is planned and, if the results are satisfactory, new programs of star observations will be started. In view of the high observational accuracy obtained with astrolabes such star observations, supplemented by additional observations of the Sun, planets, satellites and minor planets, would significantly contribute to connect the stellar and the dynamical reference systems. Observations of stars not included in the Hipparcos catalogue could help to densify and extend the optical reference frame. The justification and the realization of such plans depend, of course, strongly an the future developments in space astrometry. 
Station reports and Results: Reports were received from Santiago, Rio de Janeiro, Russia and China. Many star observations were made in China and Russia with the aim to create highly accurate catalogues of star positions. At the other stations there are no special programs for stellar observations. But work done at these stations is of significant astrometrical importance, for instance in the determination of the relation between different reference frames.

Another field of astrolabe work is the solar observations which, in cooperation with the PICARD satellite observations, provide an independent check of the space observations. In order to increase the quality of these comparisons a ground based PICARD instrument will be installed in the Calern station of the CERGA near the new DoRaySol instrument (newly automated CCD astrolabe which will begin to observe in 1999) and near the CCD solar Astrolabe (semi-automated observing continuously since 24 years, in visual and CCD mode).

Conclusion: In view of the increased capabilities of the new astrolabes and considering the large progress made in optical astrometry (e.g. Hipparcos) it is necessary to reconsider the tasks and the structure of the Astrolabe Working Group.

1) With the new generation of astrolabes and their capabilities the tasks of the WG has to be defined in a broader sense, including also cooperation with other more specialized astrometric instruments (the DoRaySol-Picard cooperation is a good example). The programme should not be restricted to star observations and catalogue construction, but it should be extended to all observable objects (not observed by space or/and interferometric methods). In particular should be included observations of the Sun, planets and other objects suited to link different reference frames.

2) A modification of the constitution of the WG should be made in order to increase the possibilities of communication and cooperation between the participating astrolabe stations. In particular a common data and software archive should be installed with free access to all members. A homogeneous set of observing data in each field of research should be made accessible to all members of the community. This needs standardisation of instruments, reduction procedures and methods of data recording.

3) For many of the stations it is still very important to have close and, in particular, officially endorsed connections to other stations in the world. In many cases such a support will help to strengthen the position of a station within its own country and thus contribute to keep alive astronomy in general.

To satisfy these objectives it is proposed to replace the "Astrolabes Working Group" by a new one specialized on CCD astrolabe astrometry of the Solar System and, in the future, also on observations of stars.

Some goals of this new working group have been described above. The new WG will include all concerned stations equipped (or near to be) with CCD cameras: CERGA, Rio de Janeiro, Sao Paulo, San Fernando, Antalya for solar observations, and Poznan and Bucarest working on new optical systems which will enable stellar observations, and, in addition, all the new ones with existing projects for installation of CCD devices at their astrolabes (e.g. Santiago).

The duration of the WG should be limited to about six years since technological progress in all fields of astrometry will give rise to soon reconsider the future of the new WG.

Based on discussions within the old WG we propose as the name of the new WG: "Alt-Azimuth CCD Astrometry" and as its president Dr. Alexandre ANDREI, who leads a very active group in Rio de Janeiro National Observatory, and who has accepted to take this responsibility. 


\section{CONFERENCES AND PROCEEDINGS}

Colloquium 165, Dynamics and Astrometry of Natural and Artificial Celestial Bodies, Poznan, Poland, July 1996, eds.: I. Wytrzyszczak, J.H. Lieske, R.A. Feldman; Kluwer (ISBN 0-7923-4574-6).

Colloquium 178, Polar Motion: Historical and Scientific Problems, Cagliari, Italy, September 1999 (in preparation).

Symposium 179, New Horizons from Multi-Wavelength Sky Surveys, Baltimore, USA, August 1996, eds.: B.J. Mc.Lean, D.A. Golombek, J.E. Hayes, H.E. Payne; Kluwer (ISBN 0-7923-4802-8).

ESA Symposium: Hipparcos-Venice '97. Isola di S. Giorgio, Italy, May 1997, B. Battrick, M.A.C. Perryman, P.L. Bernacca, K.S.O'Flaherty, ESA-SP-402, ISBN 92-9092-291-5.

Journées Systèmes de Référence Spatio-Temporales (and Lohrmann Colloquium):

Paris, Sept. 1996: Deux sciècles d'évolution du Système du Monde. ISBN 2-901057-34-2.

Prague, September 1997: Reference systems and frames in the space era: present and future astrometric programs. ISBN 2-901057-37-3.

Dresden, September 1999: Motions of Celestial Bodies, Astrometry and Astronomical Reference Frames (in preparation).

International Spring Meeting of the Astronomische Gesellschaft: The Message of the Angles - Astrometry from 1798 to 1998; eds.: P. Brosche, W.R. Dick, O. Schwarz, R. Wielen; Gotha, Germany, May 1998 (ISBN 3-8171-1588-1).

4th International Workshop on "Positional Astronomy and Celestial Mechanics", Peniscola, Spain October 1996.

Treasure-Hunting in Astronomical Plate Archives, Sonneberg, Germany, March 1999 (in preparation).

Working on the Fringe: An International Conference on Optical and IR Interferometry from Ground and Space; Dana Point CA, USA, May 1999 (in preparation).

Heiner Schwan

President of the Commission

Links: see Report of Commission 24 